\title{
Using modern informatics tools for smart decision-making in education process
}

\author{
Tadeusz Wibig, Michał Jaszczyk, Punsiri Dam-o \\ University of Lodz, Physics Dept., Pomorska 149/153, 90-236, Lodz, Poland \\ For correspondence: t.wibig@gmail.com
}

\begin{abstract}
We proposed the description of the process of evolution of the opinion of the group caused by the new information analysed. We have used the Bayesian method of the statistical inference. We found the procedure to study the change of the opinion of each individual by the influence of communication with other members of the group. We analyse also the change of the general group opinion with this mechanism. We have created the tool which uses the network of students tablets to study the effect of group work in classes. It also could conduct and control collaborative work of students. This type applets could be used further to create another new innovative tools for educational purposes.
\end{abstract}

\section{Introduction}

Famous Ash experiment shows the importance of conformity when judging even the simply and obviously clear situation. The conformity behaviour certainly works in everyday life, but we would like to believe that the scientific judgement is free of it.

The concept of modern Science is established on the objectivity of the scientific statements, experimental observations and measurements which are also assumed to be objective. We teach Science (physics in particular) trying to persuade students that the scientific facts are the same, constant, no matter who is making the judgment, the observation and analysis. In many cases we, the physicists, are proud of that, comparing, e.g., physics to the social sciences like history, which, as Napoleon said, is written by winners. This common believe of the objectivity of the Science is based on the XVII century construction of the system of empirical knowledge (or we can even go to ancient Greeks if we are here), anyway this is the basic concept of the contemporary science existing in textbooks we are using. But it seems that the end of last millennium was also the end of the era when we could say this without any doubts. The Bayesian concept of the probability based on the Bayes theorem developed already in XVIII century is going to appear in the state-of-the-art data analysis, e.g., in high-energy physics (CERN, LHC, Higgs boson searches, etc.) as well as frequentist, the classical theory. In the Review of Particle Physics of 1993 (Lynch, 1993), it is said that 'This Bayesian approach is considered unsatisfactory...'(even for two reasons), while in 2012 in the Review of Particle Physics (Beringer, 2012) concluded that for small data samples and for measurements of a parameter near a physical boundary, the Bayesian approach may yield results different then the frequentist method, and we are forced to make a choice, and no general recommendation is exists.

In the Bayesian definition of probability the subjective aspect of the creation of knowledge is expressed directly. The attempts to make the classical definition of probability objective did not go much further than the original Laplace definition for about 250 years. The number of Bayesianists grows continuously.

The Bayes theorem introduces the subjectivity to the experimental data evaluation process, but it is still the single individual involved. One's mind is using the best knowledge to estimate the truth, for 
example the value of the unknown parameter describing the reality in the most probable, the best, way, whatever it means. Of course some definition of 'the most probable way' could be given, but in principle there is no THE ONE AND ONLY definition of 'the best fit'.

It is the point which we believe should be teach, when we would like to prepare students for the former live, possibly in science, but not only. This is the general statement for the future understanding of the world around us. Methods of understanding are changing, and if we could live with the old concept, the next generations should be ready for changes

\section{The description of the process}

We would like to present here the basis of the group judgement in the Science. We will use it for parameter fitting procedure and show how the effect of the group can be found, described and analysed.

\section{Frequentist, classical way}

In the classical theory of probability for about 200 years, since the Laplace's 'Théorie analytique des probabilités' appeared, the probability has been defined as 'the ratio of the number of cases favourable to event in question, to the number of all cases possible when nothing leads us to expect that any one of these cases should occur more than any other, which renders them, for us, equally possible.'

Certainly this definition is the circulus in definiendo, the idem per idem definition: the equally probability events are needed before the probability is defined. In spite of the two centuries of tries the classical definition still exists as we gave it above. If we have developed nothing better we should accept what we already have.

If we have the theory which predict chance of the occurrence of the particular experiment result $X$, and we know about the $N$ experimental test runs with the result $X$ observed $n$ times, then we can say that the probability of the result $X$ of the future repetition of this experiment is about $n / N$. In the classical theory we cannot say that the theory if 'true' with the probability $n / N$. There is no way to define the 'probability of the theory': the theory could be only true or false. We can only estimate the chance of the result of the next, $N+1$, measurement. It is about $n / N$ chance that it will be $X$. If we perform new serial of test experiments our expectation power would increase to the square root of the respective variance, determined by $n$ and $N$.

The well-known example, the Laplace problem of the sunrise concerns the chance of the sunrise tomorrow morning. We personally could see of about 30000 sunrises, but we could assume that this occurred at least for last 6000 years - although in the Ancient Worlds exists some remarks that there were disturbances with the sun, when respective Gods and Heroes were in trouble. So, the chance that the sun will rise tomorrow (at the proper time) is of order of $(1-1 / 1500000)$. On the other hand, everyone could bet much more on that tomorrow will be usual day. This is of course the situation of today. In the Ancient World such possibility existed for real, as people believe in the Phaeton myth. Today we have the Theory. We believe that all stars in the sky follow the same law of gravity as any object around us. Observing them, we collect the experience, and we have a strong believe that the gravity will work also tomorrow. The estimation of the probability obtained above is not a good solution. Our believes are hard to estimate in the classical probability theory.

\section{The Bayesian approach}

The theory which could give the reasonable answer is the Bayesian theory of probability. Probability is defined there by a level of certainty relating to a potential outcome, and it is strongly subjective, as a 'certainty' is. The probability depends on an individual, its state, the state of its mind, so it 
obviously change with time. It evaluates. This is, in a sense, the weakness or the strength of the Bayesian approach. Individuals can learn through experience. One can bet that the particular theory is true or false, so he/she has particular degree of belief if the theory is true. It can be called by Bayesians as the probability of the theory.

The Bayes theorem is a general way describing the increase of our knowledge about the theory $T$ (the probability that it is true) by analysing the new experience (new experiment result) $X$

$$
P(T \mid X)=\frac{P(T) P(X \mid T)}{P(X)},
$$

where

- the prior estimate of probability, $P(T)$, is our initial belief about the probability of $T$ being true.

- the posterior estimate $P(T \mid X)$ is the probability of $T$ being true given that $X$ has been observed.

- the likelihood factor, $P(X \mid T)$ is the probability of event $X$ occurring if $T$ is true.

- if we consider a range of possible $T^{\prime}$ 's so we can calculate $P(X)$, the total probability of $X$ happening for any $T$.

The likelihood is subjective, because it can be calculated assuming that we know the theory T. $P(X)$ is the normalization factor, so it is also subjective, in a sense. The $P(T)$ is the factor which estimate the belief of the individual in question before the experiment begins.

In the case of tomorrow sun we have obviously the great certainty established by generations of our ancestors, and the Science which makes the subject of the question a part of the Nature, which is quite solid and rather stable.

We do not wish to go into details which can be found easy elsewhere. We would like to show clearly the starting point of our consideration.

\section{The number of individuals}

Eq.(1) works for each individual of the group $\mathbb{G}$ contain $N$, in principal similar individuals. Classically nothing is changed, but in the Bayesian way it is slightly more complicated: each individual $i$ can get different beliefs, the prior, $P_{i}(T)$, Thus the same observation $X$ with the same likelihood $P(X \mid T)$ leads $i$ to its own posteriors $P_{i}(T \mid X)$. Recording each of them, we can ask, what is average answer of the group $\mathbb{G}$. At the first approximation we can defined it as

$$
P_{\mathbb{G}}(T \mid X)=\frac{1}{N} \sum_{i=1}^{N} P_{i}(T \mid X) .
$$

With Eq.(1) we obtain

$$
P_{\mathbb{G}}(T \mid X)=\frac{1}{N} \frac{\sum_{i=1}^{N} P_{i}(T) P(X \mid T)}{P(X)}=\frac{P(X \mid T)}{P(X)} P_{\mathbb{G}}(T),
$$

where $P_{\mathbb{G}}(T)$ is the general prior the average believe that the theory $T$ is correct. The general knowledge of the group changes exactly in the same way as each individual believe. This result is the same as the conventional, frequentist, one. But it is valid only when the individuals gets its 
experimental evaluations independently using the same input $X$, and communicate only on the final stage averaging their outputs $P_{i}(T \mid X)$.

We can expect other result when group cooperate. The cooperation could be driven by different reasons, just the unconscious, instinctive or intuitive conformity as in the Ash experiment or the teamwork accepted intentionally to get the better or faster the required result, but the mechanism, theoretical description, should be unique.

The performance of Ash experiment participants exposed to the group pressure was different to performance in a control condition in which there were no confederates. The output of the confederates reported in test cases was "wrong", but this is not what we wish to describe. Their answers, posteriors, were recognized by the one genuine participant and his output was influenced by them, certainly not independent. Working as a team with the task to get the expected results, e.g., to observe the tiny, subtle effect, all claim to see it is, if only one, the first team member announce to see it.

We should modify the equation for $P_{\mathbb{G}}(T \mid X)$ to describe the collaboration (conformity) effect. If one has the prior $P_{i}(T)$ before taking part in the collaborative experiment with the output $X$, his output opinion $\mathrm{P}^{\prime}(T \mid X)$ about $T$ is influenced by the rest of the group $\mathbb{G}$. Let's denote his ability to correct his opinion as a result of the group pressure by the factor $(1-\beta)$

$$
P^{\prime}{ }_{i}(T \mid X)=\beta_{i} \cdot P_{i}(T \mid X)+\left(1-\beta_{i}\right) \cdot P_{\mathbb{G}-i}(T \mid X),
$$

where $P_{\mathbb{G}-i}(T \mid X)$ is the average output of the group without the $i$-th individual.

We are interested in the average modified group opinion $P_{\mathbb{G}}^{\prime}(T \mid X)$ as

$$
P_{\mathbb{G}}^{\prime}(T \mid X)=1 / N \sum_{i=1}^{N} P^{\prime}{ }_{i}(T \mid X) .
$$

Using definitions given above we have

$$
\begin{aligned}
& P_{\mathbb{G}}^{\prime}(T \mid X)=1 / N \sum_{i=1}^{N}\left[\beta_{i} \cdot P_{i}(T \mid X)+\left(1-\beta_{i}\right) \cdot P_{G-i}^{\prime}(T \mid X)\right]= \\
& \quad=\frac{1}{N} \sum_{i=1}^{N}\left\{\beta_{i} \cdot P_{i}(T \mid X)+\left(1-\beta_{i}\right) \cdot\left[\frac{1}{N-1} \sum_{j=1 ; j \neq i}^{N} P^{\prime}{ }_{j}(T \mid X)\right]\right\}=\cdots \text { etc. }
\end{aligned}
$$

The procedure given by Eq.(6) recursively diminish the number of elements in subsequent sum factors. If we perform it $N$ times assuming that all $\beta_{i}$ are the same as well as all individual priors, (all individuals are exactly ordinary) we obtain eventually

$$
\begin{aligned}
P^{\prime}{ }_{\mathbb{G}}(T \mid X)= & \beta \cdot P(T \mid X)+\beta(1-\beta) \cdot P(T \mid X)+\cdots \\
& \ldots+\beta \cdot(1-\beta)^{N} \cdot P(T \mid X)=P(T \mid X) \cdot\left[1-(1-\beta)^{N}\right],(7)
\end{aligned}
$$

what is different from the average opinion of the same individuals which do not communicate. The factor in brackets $[\ldots]$ is close (equal) to the unity

- if people do not communicate $(\beta=1)$,

- if number of people in the group $(N)$ is big enough (for $\beta=0.9$ and $N=10$ the difference is $10^{-9}$, for $\beta=0.5$ and $\mathrm{N}=5$ the difference is still only $3 \%$ ). 
It is interesting, that if we have the group of people which entirely do not trust their abilities $(\beta=0)$, the group could not express any opinion. If we have group of people which almost do not have their own opinion $(\beta \approx 0)$, there big number of them is need to form conclusions comparable to the opinion of the single clever, educated individual.

Another point we should notice is the fact that the factor in brackets [...] is never greater than unity. It can be concluded that there is no way to get from the group the conclusion which is stronger than the opinion of one educated man with strong self-confidence.

This conclusions are somehow intriguing (e.g., no matter how many people share the particular meaning, it could be right of wrong even if the number of believers is doubled!). The questions arises if there is any 'logical' reason why people form the group working on a particular subject together.

Of course there is an interesting problem to study, what will come out if the abilities of members of the group are not the same: if there are some with significantly different priors (higher educated!) and different degree of self-confidence, charisma, allure. This is to some extent the situation of the teacher and students. There is a distinguished individual in the group, and the effect of his behaviour could be adopted by the others according to the procedure of Eq.(6). But for the present paper we could show the example of collaboration in the group with almost equal priors and communication factors $\beta$.

\section{Parameter estimation case}

The theoretical description presented above concerns the Bayesian treatment of the general evolution of the opinion about the theory $T$, in general. More often we have to deal with the evaluation of the value of the particular parameter of the theory which we trust with no doubts. This complicate formulas, but does not change the concept. We can use the Eq.(6) in such case but changing the meaning of the $T$. If we wrote Eq.(4) in the form

$$
P^{\prime}{ }_{i}(T(t) \mid X)=\beta_{i} \cdot P_{i}(T(t) \mid X)+\left(1-\beta_{i}\right) \cdot P_{\mathbb{G}-\boldsymbol{i}}(T(t) \mid X) \quad,
$$

where $T(t)$ stands for the theory $T$ with the value of the parameter of interest equal to $t$, the meaning of the $P(T(t) \mid X)$ (or $P^{\prime}{ }_{i}(T(t) \mid X)$ ) is the probability (probability density, in general, for continuous parameter $t$ ) of the case of the theory with parameter value equal to $t$ (or in the range of $(t, t+d t))$, shortly speaking 'the probability of $t^{\prime}$.

The interpretation of such modified Eq.(6) is that it shows the change of the confidence of the result of the studies based on the opinion of the group, when we take into account the effect of influence to one member of the group by the rest of it. It is then obvious that the opinion of less self-reliant people loss its general strength.

There is an important effect of the group collaboration: the decrease of confidence. It is due to the narrowing of the spread of opinions of not self-reliant individuals. We should, in general, observe it in the real cases and, it is possible that such behaviour could be responsible for the shocking effect of Ash-type experiments.

From the educational point of view collaborative work diminish the differences between students, so it is very positive, in a sense. Dangerous effects of biasing the group opinion to the wrong direction by the erroneous behaviour of strong self-confidence individuals have to be controlled by the outer educated person - the teacher. The control is the important and it is one of the primary duties of the teacher, what is obvious. 


\section{Correlation coefficient}

Estimation of the set of parameters $\left\{\beta_{\mathrm{i}}\right\}$ is possible, in principle, but it required detailed knowledge of the distributions $P_{i}$ with respect to $t$ which could be treated in the Bayesian approach as a random variable. This knowledge can be based on the information adopted from other external sources or by the experiment itself, but this needs very big statistics what is hard to realize in practice. We found the way to simplify the model to be applied for the practical exercises.

Let's assume that we have a group of $N$ individuals (students) and, for simplicity, assume that the only one parameter describing the communication between the student is responsible for all the effect: the classical correlation coefficient between results reported by any pair of students.

$$
\rho_{i j}=\frac{\left\langle t_{i} t_{j}\right\rangle-\left\langle t_{i}\right\rangle\left\langle t_{j}\right\rangle}{\left\langle t^{2}\right\rangle-\langle t\rangle^{2}}
$$

where $t_{i}$ and $t_{j}$ are values of the parameter (e.g. Higgs particle mass) given by students $i$ and $j$. Let's further assume that all pair correlation coefficients are equal $\left(\rho_{i j}=\rho\right)$, as well as all individual $t_{i}$ estimator distributions are the same. The denominator in Eq.(9), is the variance of any (all) estimation of $t$ and it is related to the variance of the value reported by each of student in the "collaborative" experiment performed with our software. The average value of the parameter $t$ is defined for each group is

$$
\bar{t}=\frac{1}{N} \sum_{n=1}^{N} t_{n}
$$

and when the experiment is repeated $M$ times (for simplicity always with the $N$ students), we have the set of $M$ values of $\left\{\bar{t}_{m}\right\}$. They could be again treated as random variables having a respective probability density distribution, which is in quite complicated way related to each individual independent estimator distribution $t_{n}$ and to the general correlation coefficient $\rho$. Whatever elaborative is this complot, the distribution of the variable $\bar{t}$ is well defined and simple variance

$$
\operatorname{Var}(\bar{t})=\frac{\sigma_{t}^{2}}{N}(1+(N-1) \rho) .
$$

With our model the correlation coefficient has to be non-negative thus

$$
\frac{\sigma_{t}^{2}}{N} \leq \operatorname{Var}(\bar{t}) \leq \sigma_{t}^{2}
$$

with the limits of no communication at all and exact one-to-one matching of student answers.

Eq. (11) allows one to determine the correlation coefficient $\rho$ comparing variation of independent estimation $\sigma_{t}^{2}$ with the variation of the group average result $\operatorname{Var}(\bar{t})$.

\section{Collaboration in a group - an example}

The Ash experiment showed that the opinion of the member of the group could be influenced very strongly by the behaviour of the rest of the group. This, together with the conclusions of the previous section put the idea of the group work in the education process in question. If we do not want to produce individuals expressing only expected and trained conducts, what is possibly our tsk at least in teaching Science, the work in a team with no specially educated team leader (teacher) makes no advance. It is in contradiction with conventional wisdom and recipes.

We have performed the educational experiment to test the effect of communication in the group of students while solving quite complicated physical (mathematical, statistical) problem. To make the lesson more attractive we have used the extremely nowadays problem of great importance to the Physics - the Higgs boson hunting at CERN. In March the new data have been publish with the claim 'at last we have it!' (De Cecco, 2013),. The existence of 'the God particle' appears as a small bump of 
the measured rate in the observed invariant mass spectrum. The are many channels of the Higgs to decay and many plots to look at. We have taken the data from the famous picture published last summer by the CMS Collaboration (2012). The task we gave to our students is to find their fit to the Higgs peak on the graph. We made the application for tablets (with Android 4.0 or higher) with the data points plotted together with the curves of the background and Higgs peak together. The view of the screen is presented in Fig. 1. The shape and position of both curves were described by the five parameters and controlled by the five sliders beside the data plot. Students after an about half an hour lecture about the CERN, LHC and Higgs were asked to find their own Higgs particle. Up to 5 minutes takes to learn how to effectively use sliders. No special help was necessary, because children are 'digital native' and most of them start very fast and move curves in the desired directions. We have then asked them to try, as accurate as they can, to place the line showing summary of the background and the signal through the measured points. We did not explain to our students exact definition of 'the best fit', this is rather complicated task and needs time to recognize the idea of maximum likelihood or minimum $\chi^{2}$. We put attention only to avoid the common errors, for example to explain what is the background and where it should go and mistakes usually made by the amateurs, the newcomers to the statistics. The application continuously sends all actual values of the line parameters to the host tablet all the time, during the whole procedure. In the introduction explaining how the application works we mentioned that there is shown also the line presenting all the time "the result of the group" - the averaged by the host tablet actual state of all student fits. If one change its fit line (making his fit absolutely wrong) small change on the average line could been seen by others and by himself.

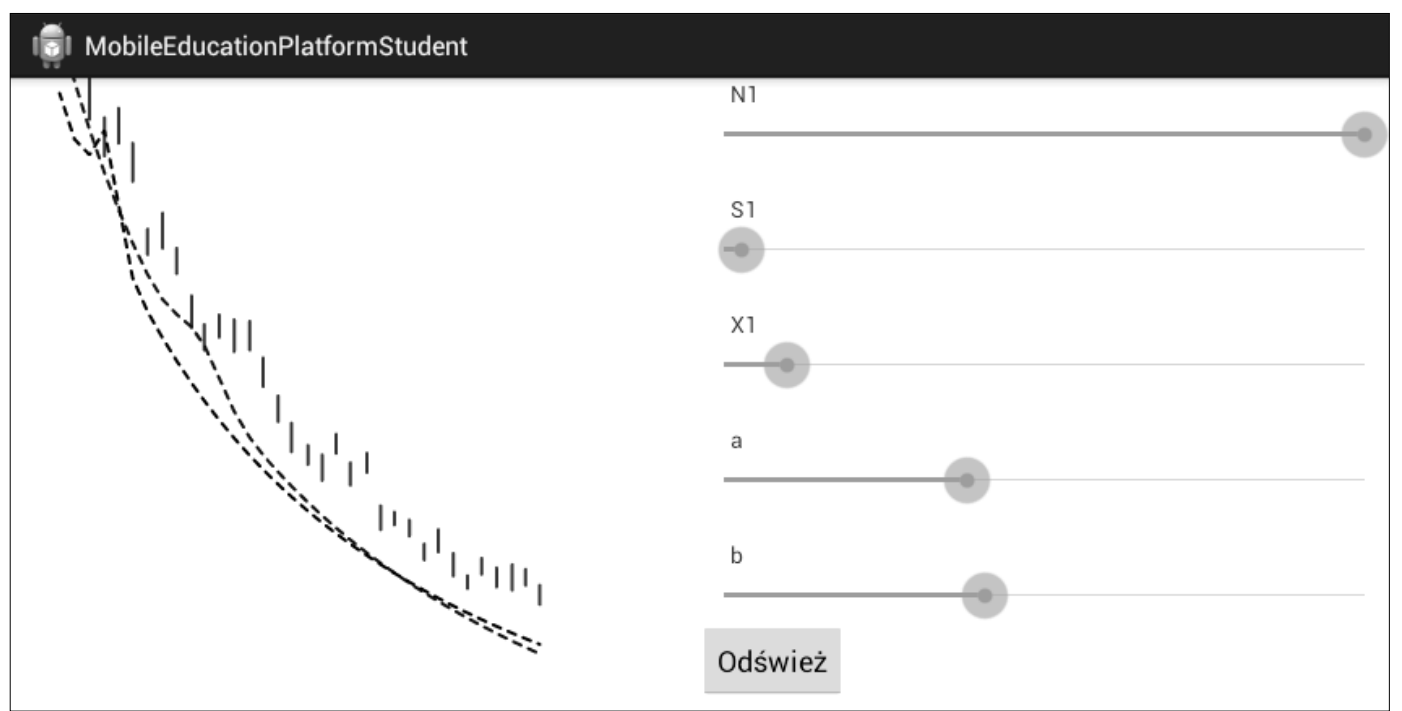

Figure 1. The view of the tablet screen. The CMS data points are represented by the small vertical (red in real) bars, the individual fit line is given by the black dashed line (the lower one in the plot). The 'average' line is the blue dashed line (upper here). Sliders are to the right.

We have asked students to perform the fit procedure not put attention to the averaged result, but it is assumed that they were able, to some extent, (consciously or not) to use this additional information, comparing all the time where they moved their line with the position preferred by others.

The schema of the Ash experiment is obvious. We wish to estimate quantitatively the communication effect. We have performed our experiments with the group of 75 students of the high schools. The all over average of the position of the Higgs particle maximum is equal to $124.75 \mathrm{GeV}$. In the original CMS paper (CMS Collaboration, 2012) the Higgs position for the $\gamma \gamma$ channel is given as $124.9 \mathrm{GeV}$ with the uncertainty of about $1 \mathrm{GeV}$. This unexpectedly big uncertainty (when we look at the data points 
trying to estimate the effect of a statistical fit procedure 'error') is due to not very clear physical picture and contaminations of other non-Higgs reaction channels.

The schema of the Ash experiment is obvious. We wish to estimate quantitatively the communication effect. We have performed our experiments with the group of 75 students of the high schools. The all over average of the position of the Higgs particle maximum is equal to $124.75 \mathrm{GeV}$. In the original CMS paper (CMS Collaboration, 2012) the Higgs position for the $\gamma \gamma$ channel is given as $124.9 \mathrm{GeV}$ with the uncertainty of about $1 \mathrm{GeV}$. This unexpectedly big uncertainty (when we look at the data points trying to estimate the effect of a statistical fit procedure 'error') is due to not very clear physical picture and contaminations of other non-Higgs reaction channels.

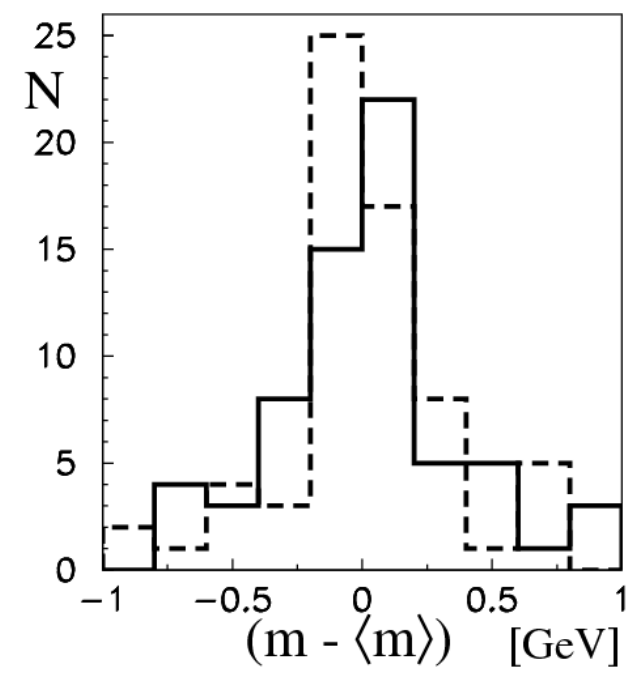

Figure 2. Distribution of Higgs mass with respect to the average mass from all fits (solid histogramindependent fits) and with respect to the respective group average (dashed histogram with communication effect taken into account).

There is also shown the histogram of the same estimated Higgs masses but with respect to the each group average mass (the dashed histogram) representing the distribution of the group estimate of $\widetilde{t}$ with the variance $\widetilde{\sigma}_{t}^{2}$. It is of course narrower. The difference is not very big what confirmed the assumption about the weak "communication effect". The difference of widths gives us the required value of the variance of the group average $\bar{t}$.

$$
\operatorname{Var}(\bar{t})=\sigma_{t}^{2}-\widetilde{\sigma}_{t}^{2}
$$

Substituting the values of widths of histograms shown in Fig.2 to Eqs.(13) and (11) we obtain the value of the correlation coefficient $\rho$ equal to $6 \%$. It is very small, but we wish to know the statistical significance of this result. It is worth to see that the distribution of estimated values are strongly nonGaussian (the relatively substantial and long tails could be results of lack of experience of students, but also a limited time we gave them to learn the new tool and, in general, quite new subject of experimental activity) and any Fisher-Snedecor like tests or ANOVA tables of critical values are not exactly applicable here. We have performed the Monte Carlo simulations to check the chance probability of the appearance of $6 \%$ or higher value of correlation coefficient with the statistics of groups and distributions we observe. We have found that the chance probability is about $30 \%$, so the observed value is just on the edge of the error box of the lack of correlation assumption.

We can say that with the experiment we performed no effect of the communication was observed. Students made their fits independently of the suggested results obtained by others members of the group. 


\section{Summary}

We proposed the description of the process of evolution of the scientific opinion of the group analysing new data. We have used the Bayesian method of the statistical inference. We found the procedure to correct the result of each individual by the influence of communication with other members of the group. We checked the possibility for modify the whole group opinion by the same mechanism. With the limited statistics the general procedure could be simplified. Introducing the one parameter describing the on-to-one correlation among student results, the common correlation coefficient, the process could be described quantitatively, and its parameter could be easy estimate experimentally.

We have created the tool to study the effect of group work in classes but also to conduct and control collaborative work of students. The application developed for our present experiment create automatic wi-fi connection with all users and turning the teachers tablet into a server. Thanks to the usage of wi-fi connection, the application can be used in school classrooms without range and connection problems. This method could be used further to create the tools for educational purposes, especially for interactive tests and much more complicated problem solving tests, training courses etc. Continuous recording of the student actions could be used to control if student works independently, when it is required, and to send immediately the alert to the teacher.

We have shown one application of the tablet network. We used it to study the effect of collaboration and communication in the group. If the effect of the group would be similar in strength to the one observed by Ash long time ago, we would be able to measure and study it. In other situations with additional accompanying factors the increase of the "collaboration needs" is expected of course and it is important to have the tool to measure it in different conditions for the group of the size of a class and further, to applied the results of such studies for educational processes. It is always good to know, which educational actions could be made more effective with the help of student intercommunications, conscious or not.

\section{References}

Beringer, J., et al. Particle Data Group, (2012) Phys. Rev. D86, 010001.

CMS Collaboration (2012) A New Boson with a Mass of $125 \mathrm{GeV}$ Observed with the CMS Experiment at the Large Hadron Collider , Science 338 , 6114, 1569-1575.

De Cecco, S., on behalf of ATLAS and CMS collaborations (2013), Higgs to di-photon in ATLAS and CMS, ATL-PHYS-SLIDE2013-191.- Geneva: CERN.

Lynch, G, (1993), Calculations of Upper Limits, PDG-93-03. 